\title{
MIR-708 promotes phagocytosis to eradicate T-ALL cells by targeting CD47
}

\author{
Wei Huang ${ }^{1}$, Wen-Tao Wang ${ }^{1}$, Ke Fang ${ }^{1}$, Zhen-Hua Chen ${ }^{1}$, Yu-Meng Sun ${ }^{1}$, Cai Han ${ }^{1}$, Lin-Yu Sun ${ }^{1}$, \\ Xue-Qun Luo ${ }^{2}$ and Yue-Qin Chen ${ }^{1,3^{*}}$
}

\begin{abstract}
Immunoevasion is a hallmark of cancer progression, and immune checkpoint blockade has emerged as a promising strategy for cancer treatment. microRNAs (miRNAs) are important negative regulators of gene expression in the immune system. Here, we demonstrate that miR-708 regulates CD47, a transmembrane protein that inhibits phagocytosis in T cell acute lymphoblastic leukemia. miR-708 directly targeted CD47 through binding to 3'UTR and is inversely correlated with CD47 expression. Functional studies showed that restoration of miR-708 expression in the T-ALL cell line is sufficient to promote phagocytosis by macrophages in the absence or presence of the anti-CD47 antibody to eradicate T-ALL cells, and inhibited tumor engraftment in vivo. Together, our findings suggest that miR-708 is a key negative regulator of CD47 and may serve as an attractive candidate for immunotherapy of T-ALL.
\end{abstract}

Keywords: miR-708, CD47, T-cell acute lymphoblastic leukemia, Targeted therapies

\section{Introduction}

T cell acute lymphoblastic leukemia (T-ALL), an aggressive hematologic tumor arising from the malignant transformation of $\mathrm{T}$ cell progenitors, accounts for approximately $15 \%$ of pediatric and $25 \%$ of adult ALL cases with high relapse rates [1]. In recent years, due to the use of chemotherapy agents, the prognosis of T-ALL has gradually improved, with a 5-year event-free survival rate reaching over $75 \%$ in children and $50 \%$ in adults [2]. However, these chemotherapy drugs are toxic and have long-term side effects, and patients with primary resistant or relapsed leukemia have poor outcomes. The limited therapeutic options available for these patients underscore the need to identify novel therapy targets and more effective antileukemia drugs [1]. Monoclonal antibodies have emerged as an attractive therapeutic modality. The properties of their target specificity, low toxicity and their ability to activate immune effector cells, such as T cells, NK cells and macrophages, make therapeutic antibodies a distinct possibility [3]. Among them, CD47 has been identified as a promising

\footnotetext{
* Correspondence: Isscyq@mail.sysu.edu.cn

${ }^{1}$ Key Laboratory of Gene Engineering of the Ministry of Education, State Key Laboratory for Biocontrol, Sun Yat-sen University, Guangzhou 510275, China ${ }^{3}$ School of Life Science, Sun Yat-sen University, Guangzhou 510275, People's Republic of China

Full list of author information is available at the end of the article
}

therapeutic antibody target in acute myeloid leukemia (AML) and acute lymphoblastic leukemia (ALL) $[4,5]$. Because the leukemia cells appear to up-regulate CD47 as a mechanism to evade the phagocytosis by macrophages, blocking the signal with the anti-CD47 antibody could specifically target leukemia cells for phagocytosis without influencing normal cells [4]. This approach is especially promising in T-ALL, since CD47 mRNA expression level is significantly higher than that of Bcells ALL or normal bone marrow [5].

Despite the beneficial effects documented for various therapeutic antibodies against different types of cancers. The antibodies are large, and they are not curative alone, possibly due to limited tissue penetration and insufficient recruitment of $\mathrm{T}$ cell effector function [6]. Methods to improve their efficacy are warranted. MicroRNAs (miRNAs), which are 20-22 nucleotides long, have important roles in cancer pathogenesis and progression since they can repress the target gene at the translational level by directly binding to the 3'untranslated regions (3'UTRs) [7]. Recent progress has been made in elucidating the roles of miRNAs in regulating immune responses as modulators of immune checkpoint molecules and their potential as cancer therapeutic targets and agents $[8,9]$. Therefore, we investigated whether 


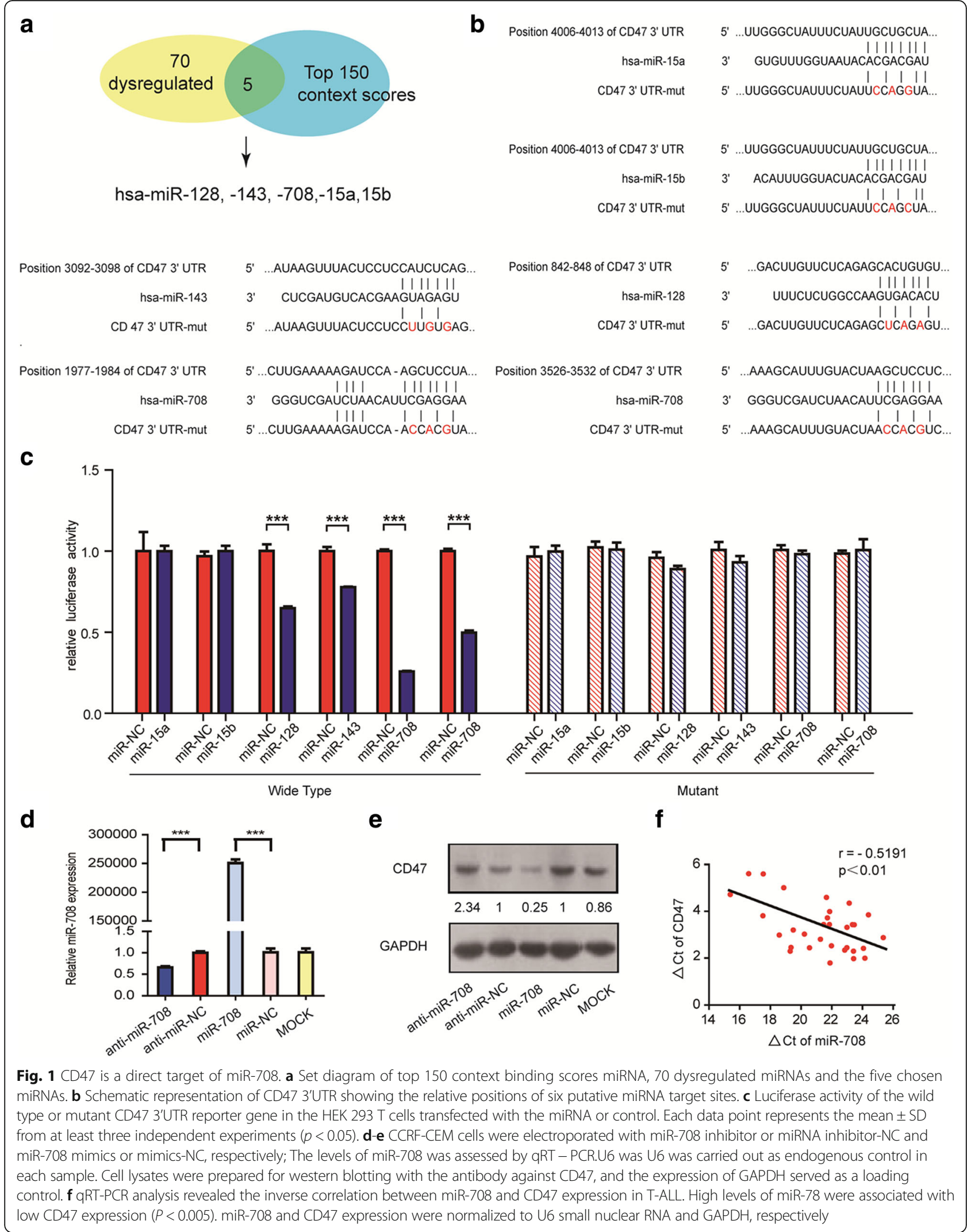


Table 1 Characteristics of test cohort

\begin{tabular}{|c|c|c|c|}
\hline Type of sample & Characteristics & Median (range) & No.(\%) \\
\hline \multirow[t]{27}{*}{ T-ALL $(N=31)$} & Age at diagnosis & $8(1-13)$ & \\
\hline & Sex & & \\
\hline & Male & & $26(83.9)$ \\
\hline & Female & & $5(16.1)$ \\
\hline & $\mathrm{WBC}, \times 10^{9} / \mathrm{L}$ & $174.8(2.75-632.47)$ & \\
\hline & Less than 20 & & $5(16.1)$ \\
\hline & 20 or higher & & $23(74.1)$ \\
\hline & N/A & & $3(9.8)$ \\
\hline & $\mathrm{FAB}$ & & \\
\hline & L1 & & $3(9.7)$ \\
\hline & L2 & & $19(61.3)$ \\
\hline & L3 & & $4(12.9)$ \\
\hline & N/A & & $5(16.1)$ \\
\hline & Risk group & & \\
\hline & $H R$ & & $17(54.8)$ \\
\hline & $\mathrm{MR}$ & & $9(29.1)$ \\
\hline & $S R$ & & 0 \\
\hline & N/A & & $5(16.1)$ \\
\hline & Prednisone response & & \\
\hline & Good respond & & $10(32.2)$ \\
\hline & Poor respond & & $16(51.6)$ \\
\hline & N/A & & $5(16.1)$ \\
\hline & Genetic mutation & & \\
\hline & MLL rearrangement & & $5(16.1)$ \\
\hline & SIL-TAL1 & & 1(3) \\
\hline & BCR-ABL1 & & 0 \\
\hline & N/A & & $25(80.9)$ \\
\hline
\end{tabular}

miRNAs could suppress CD47 to promote phagocytosis and act as miRNA-directed therapeutics.

\section{Findings}

To explore the potential regulation of CD47 by miRNAs, we performed miRNA prediction using TargetScan and re-analyzed previously published microarray data about miRNAs expression patterns in ALL [10]. Among the miRNAs with the top 150 context binding scores in TargetScan analysis, five miRNAs(miR-15a/b, miR-128, miR-143 and miR-708)with different expression patterns at diagnosis and relapse or complete remission in ALL patients were chosen for the further validation (Fig. 1a). We fused the 3'UTR sequences of CD47, each of which contained putative binding sites of these respective miRNAs (Fig. 1b), to a luciferase reporter immediately downstream from the Renilla luciferase gene. By cotransfecting the miRNA mimics with the corresponding constructs, we found that, compared with the control RNA (miR-NC, miRNA negative control), these five miRNAs showed different effects on CD47 regulation. Overexpression of miR-128 and miR-143 inhibited luciferase activity at approximately $30 \%$ and $20 \%$, respectively, while overexpressing miR-15a/b showed no effects on luciferase activity. Notably, miR-708 showed the most significant effects on luciferase activity. As shown in Fig. 1c, there were two miR-708 target sites on CD47 3'UTR, and both target sites exerted remarkable effects, which reduced the luciferase activity to approximately $75 \%$ and $50 \%$, respectively, and suggested that miR-708 is a potent inhibitor of CD47 among these five miRNAs. To further confirm the function of miR-708 upon CD47, we measured the expression level of CD47 under both miR708 overexpression and knockdown experiments in CCRF-CEM cells and examined the expression of CD47. As expected, the western blot assay showed that the protein level of CD47 was significantly reduced when CCRF-CEM cells were transfected with the miR-708 mimics, while the level of CD47 was increased by disturbing the expression of miR-708 via the miRNA inhibitor (Fig. 1d and e). We also repeated the assay on another ALL cell line, Jurkat. Transfection of miR-708 mimics significantly up-regulates the level of miR-708 and reduces the expression level of CD47 in Jurkat (Additional file 1: Figure S1A-B). This observation indicates that CD47 is the direct target of miR-708. Next, we evaluated the physiological relevance of miR-708 and CD47 by testing the expression levels of miR-708 and CD47 in clinical samples. Thirty one T-ALL patients and fifty eight B-ALL patient samples were enrolled in the study. Patient characteristics are shown in Table 1 and Additional file 2: Table S1 Spearman correlation analysis demonstrated a significant inverse correlation (Spearman $r=-0.5191 ; p<$ 0.01 ) between miR-708 abundance and the number of CD47 mRNA expression (Fig. 1f). High levels of miR-708 were associated with low CD47 expression in T-ALL. However, no negative correlation was found in B-ALL ( $p$ $=0.3052$; Additional file 1: Figure S2). These results suggested that miR-708 may function as a tumor suppressor by targeting CD47 in T-ALL.

Blocking monoclonal antibodies against CD47 has been reported to enable phagocytosis and promote apoptosis. The results above also indicated that miR-708 could suppress CD47. Thus, we assumed that forced expression of exogenous miR-708 might mimic the effects of CD47 blocking by monoclonal. Apoptosis assay was performed in CCRF-CEM and Jurkat after transfection of miR-708 mimics and mimics NC. We found that forced expression of miR-708 led to a modest increase in apoptosis rate both in CCRF-CEM and Jurkat (Additional file 1: Figure S3). We then constructed two lentivirus cell lines, named CCRF-CEM-LV-NC and CCRF-CEM-LV-miR-708, by transfecting the cells with 
a

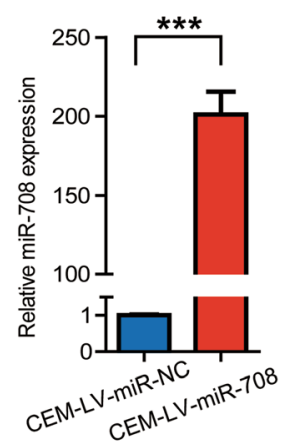

C

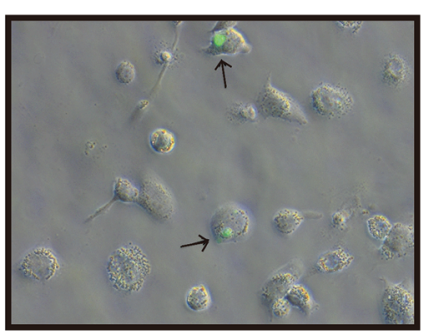

CEM-LV-miR-NC

e

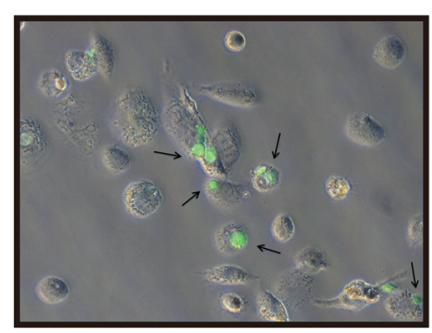

CEM-LV-miR-NC+antiCD47 antibody b

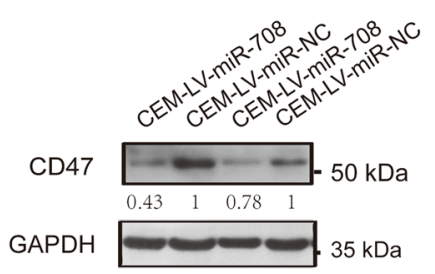

d

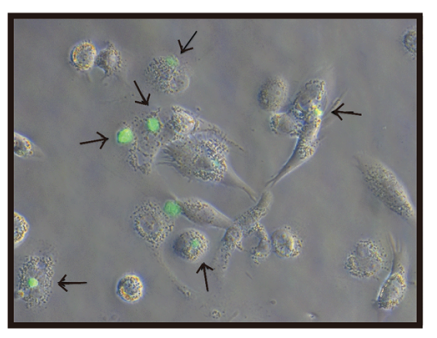

CEM-LV-miR-708

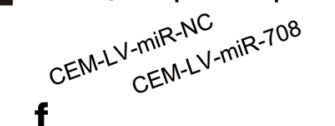

$f$

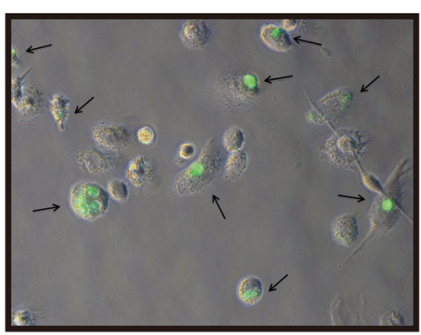

CEM-LV-miR-708+antiCD47 antibody

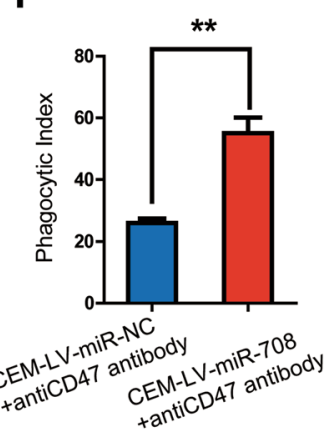

i

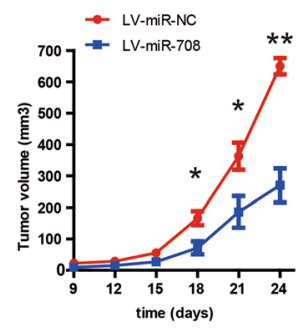

Fig. 2 Enforced expression of miR-708 enabled cell phagocytosis in vitro and inhibited tumor engraftment in vivo. a Real-time PCR ( $p<0.001)$ and (b) western blot analyses of miR-708 expression level and CD47 protein level, respectively, in CCRF-CEM-LV-NC and CCRF-CEM-LV-miR-708 cells. c. CCRF-CEM-LV-NC and CCRF-CEM-LV-miR-708 cells were fluorescently labeled green by CFSE and incubated with THP1-derived macrophages for $3 \mathrm{~h}$ and then examined by fluorescence microscopy. Arrows indicate THP1-derived macrophages containing phagocytosed CCRF-CEM cells. $\mathbf{d}$ The phagocytic index (number of target cells ingested per 100 macrophages) was determined for the indicated cell lines. Compared with the phagocytic index of CCRFCEM-LV-NC, the CCRF-CEM-LV-miR-708 shows a remarkably higher phagocytic index. e. CCRF-CEM-LV-NC and CCRF-CEM-LV-miR-708 cells were labeled in the presence of anti-CD47 antibody, incubated with THP1-derived macrophages for $3 \mathrm{~h}$ and then examined by fluorescence microscopy. $\mathbf{f}$ The phagocytic index (number of target cells ingested per100 macrophages) was determined for the indicated cell lines. The phagocytic index of CCRF-CEM-LV-miR-708 was significantly higher than that of CCRF-CEM-LV-NC $(p<0.05)$. $\mathbf{g}-\mathbf{h}$, Following the subcutaneous inoculation of CCRF-CEM-LV-NC (left) and CCRF-CEM-LVmiR-708 (right) cells into the flanks of NOD-SCID mice, overexpressed miR-708 inhibited the malignant proliferation of CCRF-CEM cells and reduced subsequent tumor size and growth (i) in vivo. Error bars reflect \pm SEM (five mice, ${ }^{*}, p<0.05$; ${ }^{*}, p<0.01$ ) 
the pCDH1-MSCV-MCS-EF1-copGFP-T2A-puro vector in which the promoter drives the expression of negative control RNA and miR-708, respectively. qRT-PCR and western blots have confirmed that miR-708 was successfully over-expressed in the CCRF-CEM cell line that was established (Fig. 2a, b). Then, we performed in vitro phagocytosis assays by incubating THP1-derived macrophages with CSFE, which is a green fluorescent dye, labeled CCRF-CEM-LV-NC and CCRF-CEM-LV-miR-708 cells and measured phagocytosis by fluorescence microscopy (Fig. 2c). As expected, we found that forced expression of miR-708 increased the phagocytosis index by approximately $20 \%(p<0.01)$. Since several studies reported that anti-CD47 antibodies enabled phagocytosis [4, 5], we hypothesized that miR-708 could promote the effects of anti-CD47 antibodies by downregulating CD47. To test this speculation, we incubated THP1-derived macrophages with CSFE-labeled CCRF-CEM-LV-NC and CCRF-CEM-LV-miR-708 cells in the presence of CD47 antibodies. Remarkably, the phagocytosis index of CCRFCEM-LV-miR-708 was almost twice that of CCRF-CEMLV-NC when only anti-CD47 antibody was added (Fig. 2d), which indicates that $\mathrm{CD} 47$ antibodies synergizing with miR-708 could serve as a potent therapeutic method against T-ALL. We further performed in vivo experiment in a NOD/SCID xenograft mouse model (methods see Additional file 3). CCRF-CEM cells transfected with LVmiR-708 were subcutaneously implanted in NOD/SCID mice. As shown in Fig. 2g-i and Additional file 1: Figure S4, overexpression of miR-708 inhibited the malignant proliferation of T-ALL tumors. These results supported miR-708-mediated pathogenesis in targeting CD47 to suppress leukemia progression.

\section{Conclusion}

We identified a novel mechanism through which miR708 regulates the CD47 immune checkpoint in T-ALL. miR-708 directly binds the 3'UTR of CD47 and inhibits CD47 expression. The inverse correlation of miR-708 and CD47 in T-ALL patients indicated a tumor suppressor role for miR-708. Indeed, restoration of miR-708 expression could promote phagocytosis to eradicate T-ALL cells in vitro, and inhibited tumor engraftment in vivo. Furthermore, combination of MIR-708 and CD47 antibodies caused a greater phagocytosis activity of macrophages on CEM cells than either agent alone. This study suggests that miR-708 is a potent regulator of CD47 and may be used to optimize anti-leukemia therapy.

\section{Additional files}

Additional file 1: Figure S1. (A-B). Jurkat cells were electroporated with mimics-NC and mimics-miR-708, The levels of miR-708 was assessed by qRT - PCR and normalized to U6.Cell lysates were prepared for western blotting with the antibody against CD47, and the expression of GAPDH served as a loading control. Figure S2. qRT-PCR analysis of the expressoion of miR708 and CD47 in B-ALL. U6 and GAPDH were used as endogenous control. Figure S3. Apoptosis assay of CCRF-CEM and Jurkat upon transfection of miR-708 mimics or mimics-NC, respectively. Figure S4. Following the subcutaneous inoculation of CCRF-CEM-LV-NC and CCRF-CEM-LV-miR-708, the levels of miR-708 and CD47 were assessed by qRT - PCR and western blot, respectively.(A-B). Overexpressed miR-708 reduced tumor weight. Error bars reflect \pm SEM (five mice, ${ }^{*}, p<0.05 ; * *, p<0.01$ ).(C). (DOCX $30259 \mathrm{~kb}$ )

Additional file 2: Table S1. Characteristics of test cohort. (DOCX $14 \mathrm{~kb}$ ) Additional file 3: Materials and methods. (DOCX $22 \mathrm{~kb}$ )

\section{Abbreviations}

3'UTR: 3' untranslated region; ALL: Acute lymphoblastic leukemia; AML: Aacute myeloid leukemia; B-ALL: B cell acute lymphoblastic leukemia; miRNA: microRNA; miR-NC: miRNA negative control; T-ALL: T cell acute lymphoblastic leukemia

\section{Acknowledgements}

This research was supported by National Key R\&D Program of China (No. 2017YFA0504400) and National Natural Science Foundation of China (No. 81770174 and 31700719),and grants from Guangdong Province (No. 2014 T70833).

\section{Authors' contributions}

WH designed and performed the research, analyzed data and wrote the manuscript. WTW, KF, ZHC, YMS, LYS and CH performed the research and analyzed data. XQL collected and analyzed clinical data. YQC designed the research and wrote the manuscript. All authors read and approved the final manuscript.

\section{Ethics approval and consent to participate}

The study was approved by the ethics committee of the affiliated hospitals of Sun Yat-sen University.

\section{Consent for publication}

Not applicable.

\section{Competing interests}

The authors declare that they have no competing interests.

\section{Publisher's Note}

Springer Nature remains neutral with regard to jurisdictional claims in published maps and institutional affiliations.

\section{Author details}

${ }^{1}$ Key Laboratory of Gene Engineering of the Ministry of Education, State Key Laboratory for Biocontrol, Sun Yat-sen University, Guangzhou 510275, China. ${ }^{2}$ The First Affiliated Hospital of Sun Yat-sen University, Guangzhou 510080, China. ${ }^{3}$ School of Life Science, Sun Yat-sen University, Guangzhou 510275, People's Republic of China.

Received: 1 September 2017 Accepted: 15 January 2018

Published online: 24 January 2018

References

1. Van Vlierberghe P, Ferrando A. The molecular basis of T cell acute lymphoblastic leukemia. J Clin Invest. 2012;122:3398-406.

2. Pui CH, Robison LL, Look AT. Acute lymphoblastic leukaemia. Lancet. 2008;371:1030-43.

3. Weiner LM, Surana R, Wang S. Monoclonal antibodies: versatile platforms for cancer immunotherapy. Nat Rev Immunol. 2010;10:317-27.

4. Majeti R, Chao MP, Alizadeh AA, Pang WW, Jaiswal S, Gibbs KJ, van Rooijen N, Weissman IL. CD47 is an adverse prognostic factor and therapeutic antibody target on human acute myeloid leukemia stem cells. Cell. 2009;138:286-99.

5. Chao MP, Alizadeh AA, Tang C, Jan M, Weissman-Tsukamoto R, Zhao F, Park CY, Weissman IL, Majeti R. Therapeutic antibody targeting of CD47 eliminates human acute lymphoblastic leukemia. Cancer Res. 2011;71:1374-84.

6. Chan AC, Carter PJ. Therapeutic antibodies for autoimmunity and inflammation. Nat Rev Immunol. 2010;10:301-16. 
7. Esquela-Kerscher A, Slack FJ. Oncomirs - microRNAs with a role in cancer. Nat Rev Cancer. 2006;6:259-69.

8. Chen L, Gibbons DL, Goswami S, Cortez MA, Ahn YH, Byers LA, Zhang X, Yi $X$, Dwyer D, Lin W, et al. Metastasis is regulated via microRNA-200/ZEB1 axis control of tumour cell PD-L1 expression and intratumoral immunosuppression. Nat Commun. 2014;5:5241.

9. Wei J, Nduom EK, Kong LY, Hashimoto Y, Xu S, Gabrusiewicz K, Ling X, Huang N, Qiao W, Zhou S, et al. MiR-138 exerts anti-glioma efficacy by targeting immune checkpoints. Neuro-Oncology. 2016;18:639-48.

10. Han BW, Feng DD, Li ZG, Luo XQ, Zhang H, Li XJ, Zhang XJ, Zheng LL, Zeng $\mathrm{CW}$, Lin $\mathrm{KY}$, et al. A set of miRNAs that involve in the pathways of drug resistance and leukemic stem-cell differentiation is associated with the risk of relapse and glucocorticoid response in childhood ALL. Hum Mol Genet. 2011;20:4903-15.

Submit your next manuscript to BioMed Central and we will help you at every step:

- We accept pre-submission inquiries

- Our selector tool helps you to find the most relevant journal

- We provide round the clock customer support

- Convenient online submission

- Thorough peer review

- Inclusion in PubMed and all major indexing services

- Maximum visibility for your research

Submit your manuscript at www.biomedcentral.com/submit
Biomed Central 